\title{
Some Effects on Quantum Systems due to the Gravitational Field of a Topological Defect
}

\author{
Geusa de A. Marques ${ }^{1}$ and V. B. Bezerra ${ }^{2}$ \\ 1. Departamento de Física, Universidade Federal de \\ Campina Grande, 58109-790, Campina Grande, Pb, Brazil \\ 2. Departamento de Física, Universidade Federal da Paraíba, \\ 58051-970, João Pessoa, Pb, Brazil \\ (Received on 14 October, 2005)
}

\begin{abstract}
We study the behavior of a non-relativistic quantum particle interacting with different potentials, in the background space-time generated by a cosmic string. We find the energy spectra for the quantum systems under consideration and discuss how they differ from their flat Minkowski space-time values.
\end{abstract}

The study concerning the influence of potentially observable effects of gravitational fields at the atomic level has been an exciting research field. Along this line of research the hydrogen atom, for example, has been studied in different gravitational fields[1-3]. These studies considered a problem which suggests potentially observable effects of gravitational fields at atomic level and showed that an atom placed in a gravitational field is influenced by its interaction with the local curvature as well as with the topology of the space-time, and as a consequence there is a shift in the energy of each atomic level which depends on the features of the space-time. The problem of finding these shifts [4] in the energy levels under the influence of gravitational fields is of considerable theoretical as well as observational interest.

The general theory of relativity, predicts that gravitation is manifested as the curvature of space-time which is characterized by the Riemann curvature tensor. Therefore, it seems interesting to know how the curvature of space-time at the position of the atom affects its spectrum. On the other hand, we know that there are connections between topological properties of the space and its local intrinsic geometry, and therefore it is not possible to describe completely the physics of a given system based only on its geometrical features. Thus, it is also important to investigate the role played by the topology on a given physical system. Therefore, the problem of finding how the energy spectrum of an atom is perturbed by a gravitational field has to take into account the geometrical and topological features of the considered space-time as well.

As examples of the influence of the topology on an atomic system, we can mention the studies concerning to quantum mechanics on a cone[5-7], the interaction of a quantum system with space-times generated by topological defects $[8$, 9] and the modification of the energy levels of a hydrogen atom[3] placed in the gravitational field of a cosmic string[10].

The space-time of a cosmic string is quite remarkable: its geometry is flat everywhere apart from the symmetry axis. The space-time around a cosmic string is locally flat but not globally. Thus, the external gravitational field due to a cosmic string[11] may be described by a commonly called conical geometry. As a consequence of this conical structure, a particle placed at rest around a straight, infinite, static string will no be attracted to it. Therefore, there is no local gravity in the space surrounding a cosmic string. Also there is another effect produced in this background, namely, the shifts in the energy levels of a hydrogen atom[3], which corresponds to the gravitational analogue[12] of the well known electromagnetic Aharonov-Bohm effect.

We will concerning the effects of the nontrivial topology of the space-time generated by a cosmic string at the atomic level. In order to investigate this problem, we consider the question of how the shifts in the energy spectrum of a particle are when it experiences different potentials, like the generalized Kratzer and Morse potentials in the space-time of a cosmic string.

In what follows we will determine the energy spectrum of a non-relativistic quantum particle interacting with a Kratzer potential and in the presence of the gravitational field of a cosmic string.

In order to do this, let us assume that a non-relativistic particle existing in a curved space-time is described by the Schrödinger equation which reads

$$
i \hbar \frac{\partial \Psi}{\partial t}=-\frac{\hbar^{2}}{2 \mu} \nabla_{L B}^{2} \Psi+V \Psi
$$

where $\nabla_{L B}^{2}=g^{-1 / 2} \partial_{i}\left(g^{i j} g^{1 / 2} \partial_{j}\right)$, with $i, j=1,2,3$ is the Laplace-Beltrami operator, $g=\operatorname{det}\left(g_{i j}\right), \mu$ is the mass of the particle and $V$ is the external potential experienced by it.

The line element corresponding to the cosmic string spacetime is given, in spherical coordinates[10], by

$$
d s^{2}=-d t^{2}+d r^{2}+r^{2} d \theta^{2}+\alpha^{2} r^{2} \sin ^{2} \theta d \varphi^{2} .
$$

The parameter $\alpha=1-4 G \bar{\mu}$ runs in the interval $(0,1]$, with $\bar{\mu}$ being the linear mass density of the cosmic string (In this paper we will consider $c=1$ ).

The Schrödinger equation in this background can be separated as

$$
\left[\frac{-\hbar^{2}}{2 \mu r^{2}} \frac{d}{d r}\left(r^{2} \frac{d}{d r}\right)-\frac{\lambda \hbar^{2}}{2 \mu r^{2}}+V(r)\right] R(r)=E R(r)
$$

and

$$
\frac{1}{\sin \theta} \frac{d}{d \theta}\left(\sin \theta \frac{d \Theta}{d \theta}\right)-\frac{m^{2}}{\alpha^{2} \sin ^{2} \theta} \Theta-\lambda \Theta=0
$$


where $\lambda$ is a separation constant and we have used that

$$
\Psi(r, \theta, \varphi, t)=R(r) \Theta(\theta) e^{i m \varphi} e^{-i E t / \hbar},
$$

where $m$ assumes the values $m=0, \pm 1, \pm 2, \pm 3 \ldots \ldots$

The solution of the Eq. (4) can be written in the form

$$
\begin{aligned}
F_{l_{(\alpha)}}^{m_{(\alpha)}}(\cos \theta)= & P_{l_{(\alpha)}^{m_{(\alpha)}}(\cos \theta)=} \\
& \sqrt{\frac{2}{\pi} \frac{(1-\cos \theta)^{\frac{1}{2}}}{\Gamma\left(\frac{1}{2}-m_{(\alpha)}\right)}} \\
& \times{ }_{0}^{\mathrm{Z} \arccos (\cos \theta)} \frac{\cos \left[\left(l_{(\alpha)}+\frac{1}{2}\right) t\right]}{(\cos t-\cos \theta)^{m_{(\alpha)}+\frac{1}{2}}} d t
\end{aligned}
$$

with $P_{l_{(\alpha)}}^{m_{(\alpha)}}(\cos \theta)$ being the generalized associated Legendre functions and $l_{(\alpha)}=\frac{m}{\alpha}+n \equiv m_{(\alpha)}+n=l-\left(1-\frac{1}{\alpha}\right) m ; l=$ $0,1,2, \ldots$.

Now, let us turn to the radial equation which can be written as

$$
-\frac{\hbar^{2}}{2 \mu} \frac{d^{2} u(r)}{d r^{2}}+V(r)_{e f f .} u(r)=E u(r),
$$

where $u(r)=r R(r)$ and

$$
V(r)_{e f f .}=V(r)-\frac{\lambda}{r^{2}}
$$

is the effective potential.

Considering the potential in the Eq. (8) as being given by

$$
V(r)=-2 D\left(\frac{A}{r}-\frac{1}{2} \frac{A^{2}}{r^{2}}\right)
$$

which corresponds to the Kratzer potential, where $A$, and $D$ are positive constants, substituting Eq. (9) into (7) and using the fact that $\lambda=-\hbar^{2} l_{(\alpha)}\left(l_{(\alpha)}+1\right) / 2 \mu$, we obtain the following solution

$$
u(r)={ }_{1} F_{1}\left(\frac{1}{2}+\frac{1}{2} \sqrt{1+4 P}-\frac{\mu}{\beta \hbar^{2}}, 1+\sqrt{1+4 P} ; 2 \beta r\right)
$$

where ${ }_{1} F_{1}$ is a confluent hypergeometric function, $P=$ $l_{(\alpha)}\left(l_{(\alpha)}+1\right)+2 \frac{\mu A^{2} D}{\hbar^{2}}$ and $\beta^{2}=-\frac{2 \mu E}{\hbar^{2}}$

This solution diverges, unless

$$
\begin{aligned}
& \frac{1}{2}+\frac{1}{2} \sqrt{1+4\left(l_{(\alpha)}\left(l_{(\alpha)}+1\right)+2 \frac{\mu A^{2} D}{\hbar^{2}}\right)} \\
& -\frac{\mu}{\beta \hbar^{2}}=-n^{\prime} ; n^{\prime}=0,1,2 \ldots
\end{aligned}
$$

Then, from this condition we find the energy eigenvalues

$$
\begin{aligned}
& E_{n^{\prime}}=-\frac{2 D^{2} A^{2} \mu}{\hbar^{2}} \\
& \times\left[\frac{1}{2}+\frac{1}{2} \sqrt{1+4 l_{(\alpha)}\left(l_{(\alpha)}+1\right)+8 \frac{\mu A^{2} D}{\hbar^{2}}}+n^{\prime}\right]^{-2}
\end{aligned}
$$

In order to estimate the effect of the presence of the cosmic string on the energy shifts, we will take $\alpha \cong 0.999999$, which corresponds to a GUT cosmic string. In this case, the shifts in the energy spectrum of the particle in the presence of the cosmic string, corresponding to the first two levels, decreases about $4 \times 10^{-3} \%$, as compared with the values of the flat Minkowski space-time. This means that the difference in the energy between the first excited state and the ground state of a hydrogen atom is very small and corresponds to a decrease in the wavelength for a transition between these two states of about $10^{-3} \%$ as compared to the flat Minkowski space-time value.

Now, we will consider the Morse potential. It can be written as

$$
V(r) \cong-D+\frac{1}{2} M \omega^{2}\left(r-r_{0}\right)^{2}
$$

Introducing the variable $r-r_{0}=r^{\prime}$, it can be written as

$$
V\left(r^{\prime}\right) \cong-D+\frac{1}{2} M \omega^{2} r^{\prime 2}
$$

This form of the potential is similar to the one which corresponds to the isotropic harmonic oscillator plus a constant term. Thus, the radial part of the Schrödinger equation is

$$
\begin{aligned}
& -\frac{\hbar^{2}}{2 M} \frac{d^{2} g\left(r^{\prime}\right)}{d r^{\prime 2}}+\left(V\left(r^{\prime}\right)+\frac{\hbar^{2}}{2 M} l_{(\alpha)} \frac{\left(l_{(\alpha)}+1\right)}{r^{\prime 2}}\right) g\left(r^{\prime}\right) \\
= & E g\left(r^{\prime}\right),
\end{aligned}
$$

where we have considered

$$
\psi\left(r^{\prime}, \theta, \varphi\right)=\frac{g\left(r^{\prime}\right)}{r^{\prime}} \Theta(\theta) \phi(\varphi) .
$$

This equation can also be rewritten as

$$
\begin{aligned}
& \frac{d^{2} g\left(r^{\prime}\right)}{d r^{\prime 2}}-\frac{M^{2} \omega^{2} r^{\prime 2}}{\hbar^{2}} g\left(r^{\prime}\right)-l_{(\alpha)} \frac{\left(l_{(\alpha)}+1\right)}{r^{\prime 2}} g\left(r^{\prime}\right) \\
& +\frac{2 M}{\hbar^{2}} E g\left(r^{\prime}\right)=0
\end{aligned}
$$

where

$$
g\left(r^{\prime}\right)=\exp \left(-\frac{1}{2} M \frac{\omega}{\hbar} r^{\prime 2}\right) r^{\prime \frac{1}{2}+\frac{1}{2} \sqrt{1+4 l_{(\alpha)}\left(l_{(\alpha)}+1\right)}} F\left(r^{\prime}\right) .
$$

Substituting (17) into (16), we find

$$
\begin{aligned}
& r^{\prime} \frac{d^{2} F\left(r^{\prime}\right)}{d r^{\prime 2}}+\left[c-\frac{2 M \omega}{\hbar} r^{\prime 2}\right] \frac{d F\left(r^{\prime}\right)}{d r^{\prime}} \\
& +\left[Q-\frac{2 M \omega}{\hbar}\right] r^{\prime} F\left(r^{\prime}\right)=0,
\end{aligned}
$$


where

$$
\begin{aligned}
c & =1+\sqrt{1+4 l_{(\alpha)}\left(l_{(\alpha)}+1\right)} ; \\
Q & =\frac{2 M E_{M}}{\hbar^{2}}-\frac{M \omega}{\hbar} \sqrt{1+4 l_{(\alpha)}\left(l_{(\alpha)}+1\right)}-\frac{2 M D}{\hbar^{2}} .
\end{aligned}
$$

The solution of Eq. (18) is

$$
\begin{aligned}
F\left(r^{\prime}\right)= & { }_{1} F_{1}\left(\frac{1}{2}-\frac{E}{2 \hbar \omega}+\frac{D}{2 \hbar \omega}\right. \\
& +\frac{1}{4} \sqrt{1+4 l_{(\alpha)}\left(l_{(\alpha)}+1\right)}, \\
& \left.\frac{1}{2}+\frac{1}{2} \sqrt{4 l_{(\alpha)}\left(l_{(\alpha)}+1\right)} ; \frac{M \omega r^{\prime 2}}{\hbar}\right),
\end{aligned}
$$

which is divergent, unless the following condition is fulfilled

$$
\begin{aligned}
\frac{1}{2}-\frac{E}{2 \hbar \omega}+\frac{D}{2 \hbar \omega}+\frac{1}{4} \sqrt{1+4 l_{(\alpha)}\left(l_{(\alpha)}+1\right)} & =-n ; \quad(20) \\
n & =0,1,2,3, \ldots .
\end{aligned}
$$

This condition gives us the following result for the energy spectrum

$$
E=\hbar \omega\left[N_{l}+\frac{3}{2}\right]+D
$$

where

$$
N_{l}=\frac{1}{2}\left(\sqrt{1+4 l_{(\alpha)}\left(l_{(\alpha)}+1\right)}-1\right)+2 n_{M}
$$

An estimation of the shift in the energy levels for this case, shows that there is a decrease in the energies of about $10^{-5} \%$ for GUT cosmic strings as compared to the flat space-time corresponding value.

It is worth commenting that in the two cases considered the obtained results tell us that the energy spectra are modified as compared to the flat Minkowski space-time result and these shifts are connected with the conical structure of the spacetime generated by a cosmic string. In other words, these shifts in the energies are due completely to the topological features of this space-time.

To end up, let us comment that the study of a quantum system in a non-trivial gravitational background, like in the space-time of a cosmic string may shed some light on the problems of combining quantum mechanics and general relativity.

\section{Acknowledgments}

We acknowledge Conselho Nacional de Desenvolvimento Científico e Tecnológico $(\mathrm{CNPq})$ and Coordenação de Aperfeiçoamento de Pessoal de Nível Superior (CAPES) Programa PROCAD and CNPq/FAPESQ-Pb(PRONEX) for partial financial support.
[1] J. Audretsch and G. Schäffer, Gen. Rel. Grav. 9, 243 (1978); 9, 489 (1978)

[2] L. Parker, Phys. Rev. Lett. 44, 1559 (1980).

[3] Geusa de A. Marques and Valdir B. Bezerra, Phys. Rev. D66, 105011 (2002).

[4] L. Parker and L. Pimentel, Phys. Rev. D44, 3180 (1982).

[5] P. de Sousa Gerbert and R. Jackiw, Commun. Math. Phys. 124, 229 (1989).

[6] M. Alvarez, J. Phys. A32, 4079 (1999); J. Spinelly, E. R. Bezerra de Mello, and V. B. Bezerra, Class. Quantum Grav. 18, 1555 (2001).

[7] V. B. Bezerra, Class. Quantum Grav. 8, 1939 (1991); E. S. Moreira, Phys. Rev. A58, 1678 (1998); Cláudio Furtado and Fer- nando Moraes, J. Phys. A33, 5513 (2000).

[8] G. 'tHooft, Commun. Math. Phys. 117, 685 (1988).

[9] Boryard S. Kay and Urban M. Studer, Commun. Math. Phys. 139, 103 (1991).

[10] Geusa de A. Marques and V. B. Bezerra, Mod. Phys. Lett. A14, 2687 (1999); Class. Quantum Grav. 19, 985 (2002).

[11] A. Vilenkin, Phys. Rev. D23, 852 (1981); B. Linet, Gen. Rel. Grav. 17, 1109 (1985); J. R. Gott III, Astrophys. J. 288, 422 (1985).

[12] L. H. Ford and A. Vilenkin, J. Phys. A14, 2353 (1981); V. B. Bezerra, Phys. Rev. D35, 2031 (1987); Ann. Phys. (NY), 203, 392 (1990). 